\title{
ON SOME PROPERTIES OF INTUITIONISTIC FUZZY SOFT BOUNDARY
}

\author{
Sabir HUSSAIN \\ Department of Mathematics, College of Science, Qassim University, P.O. Box 6644, Buraydah \\ 51482, SAUDI ARABIA
}

\begin{abstract}
The purpose of this paper is to initiate the concept of Intuitionistic Fuzzy(IF) soft boundary. We discuss and explore the characterizations and properties of IF soft boundary in general as well as in terms of IF soft interior and IF soft closure. Examples and counter examples are also presented to validate the discussed results.
\end{abstract}

\section{INTRODUCTION}

The notion of fuzzy sets was introduced by Zadeh [23]. After that several researches were conducted on the generalizations of the notion of fuzzy set. As a generalization of the notion of fuzzy set, intuitionistic fuzzy set (IFS) and intuitionistic L-fuzzy sets (ILFS) were initiated and explored by Atanassov [1-3] and [5].

In our daily life situations, we usually face complicated problems in different fields like economics, engineering, medical sciences, social sciences, etc. involving imprecise and uncertain data in nature. Uncertainties cannot be handled using traditional mathematical tools but may be dealt with using a wide range of existing theories such as the probability theory, the theory of (intuitionistic) fuzzy sets, the theory of vague sets, the theory of interval mathematics, and the theory of rough sets. However, all of these have their advantages as well as inherent limitations in dealing with uncertainties. One major problem shared by those theories is their incompatibility with the parameterization tools. To overcome these difficulties, Molodtsov [19] introduced the concept of soft set as a new mathematical tool for dealing with uncertainties that is free from the difficulties that have troubled the

2020 Mathematics Subject Classification. 54A05, 54A20, 54C08, 54D10.

Keywords and phrases. IF sets, IF soft sets, IF soft topology, IF soft interior(closure), IF soft boundary.

\sabiriub@yahoo.com; sh.hussain@qu.edu.sa

(D) 0000-0001-9191-8172.

(C)2020 Ankara University Communications Faculty of Sciences University of Ankara-Series A1 Mathematics and Statistics 
usual theoretical approaches. Molodtsov pointed out several directions for the applications of soft sets. This theory has proven useful in many different fields such as decision making [6][20], data analysis [4][24], forecasting [21] and so on. The topological structures of soft sets are studied and discussed in [7-13].

Maji et al. introduced the concept of intuitionistic fuzzy soft sets[16-18], which is a generalization of fuzzy soft sets[15] and standard soft sets. It is to be noted that the parameters may not always be crisp, rather they may be intuitionistic fuzzy in nature. The problems of object recognition have received paramount importance in recent years. The recognition problem may be viewed as multiobserver decision making problem, where the final identification of the object is based on the set of inputs from different observers who provide the overall object characterization in terms of diverse set of parameters. Different algebraic structures of IF soft sets are studied and explored in [22]. D. Coker [5] introduced and studied the concept of IF topological spaces. Z. Li et.al [14] initiated IF topological structures of IF soft sets. They explored the notions of IF soft open(closed) sets, IF soft interior(closure) and IF soft base in IF soft topological spaces.

In this paper, we initiate the concept of IF soft boundary. We discuss and explore the characterizations and properties of IF soft boundary in general as well as in terms of IF soft interior and IF soft closure. Examples and counter examples are also presented to validate the discussed results.

\section{Preliminaries}

First we recall some definitions and results which will use in the sequel.

Definition 1. 23] A fuzzy set $f$ on $X$ is a mapping $f: X \rightarrow I=[0,1]$. The value $f(x)$ represents the degree of membership of $x \in X$ in the fuzzy set $f$, for $x \in X$.

Definition 2. 19] Let $X$ be an initial universe and $E$ be a set of parameters. Let $P(X)$ denotes the power set of $X$ and $A$ be a non-empty subset of $E$. A pair $(F, A)$ is called a soft set over $X$, where $F$ is a mapping given by $F: A \rightarrow P(X)$. In other words, a soft set over $X$ is a parameterized family of subsets of the universe $X$. For $e \in A, F(e)$ may be considered as the set of e-approximate elements of the soft $\operatorname{set}(F, A)$.

Definition 3. 15] Let $I^{X}$ denotes the set of all fuzzy sets on $X$ and $A \subseteq X$. $A$ pair $(f, A)$ is called a fuzzy soft set over $X$, where $f: X \rightarrow I^{X}$ is a function. That $i s$, for each $a \in A, f(a)=f_{a}: X \rightarrow I$ is a fuzzy set on $X$.

Definition 4. 2] An intuitionistic fuzzy set $A$ over the universe $X$ is defined as: $A=\left\{\left(x, \mu_{A}(x), \nu_{A}(x)\right) ; x \in X\right\}$,

where $\mu_{A}: X \rightarrow[0,1], \nu_{A}: X \rightarrow[0,1]$ with the property that $0 \leq \mu_{A}(x)+$ $\nu_{A}(x) \leq 1$, for all $x \in X$. The values $\mu_{A}(x)$ and $\nu_{A}(x)$ represent the degree of membership and nonmembership of $x$ to $A$ respectively . 
Definition 5. 2] Let $A=\left\{\left(x, \mu_{A}(x), \nu_{A}(x)\right) ; x \in X\right\}$ and $B=\left\{\left(x, \mu_{B}(x), \nu_{B}(x)\right) ; x \in X\right\}$ are intuitionistic fuzzy set over the universe $X$. Then

(1) $A^{c}=\left\{\left(x, \nu_{A}(x), \mu_{A}(x)\right) ; x \in X\right\}$.

(2) $A \subseteq B$ if and only if $\mu_{A}(x) \leq \mu_{B}(x)$ and $\nu_{A}(x) \geq \nu_{B}(x)$, for all $x \in X$.

(3) $A=B$ if and only if $A \subseteq B$ and $B \subseteq A$.

(4) $A \cap B=\left\{\left(x, \min \left\{\mu_{A}(x), \mu_{B}(x)\right\}, \max \left\{\nu_{A}(x), \nu_{B}(x)\right\}: x \in X\right\}\right.$.

(5) $A \cup B=\left\{\left(x, \max \left\{\mu_{A}(x), \mu_{B}(x)\right\}, \min \left\{\nu_{A}(x), \nu_{B}(x)\right\}: x \in X\right\}\right.$.

Definition 6. 22 An intuitionistic fuzzy set $A$ over the universe $X$ is said to be intuitionistic fuzzy null set denoted as $\tilde{0}$, and is defined as: $A=\{(x, 0,1): x \in X\}$.

Definition 7. 2] An intuitionistic fuzzy set $A$ over the universe $X$ is said to be intuitionistic fuzzy absolute set denoted as $\tilde{1}$, and is defined as: $A=\{(x, 1,0): x \in$ $X\}$.

Definition 8. 17] Let $X$ be the initial universal set and $E$ be the set of parameters. Let $I F^{X}$ denotes the set of all intuitionistic fuzzy soft sets on $X$ and $A \subseteq X$. A pair $(I F, A)$ is called a IF fuzzy soft set over $X$, where $f: A \rightarrow I F^{X}$ is a function. That is, for each $a \in A, f(a)=f_{a}: A \rightarrow I F^{X}$, is an intuitionistic fuzzy set on $X$ and is defined as: $F(a)=\left\{\left(x, \mu_{A}(x), \nu_{A}(x)\right) ; x \in X\right\}$.

From now on, for our convenience, we will represent the intuitionistic fuzzy soft set (IF, A) as IF soft set $f_{A}$. Now we give the example of intuitionistic fuzzy soft sets as:

Example 9. Let $(I F, A)=f_{A}$ describe the character of the employees with respect to the given parameters, for finding the best employee of the financial year. Let the set of employees under consideration be $X=\left\{x_{1}, x_{2}, x_{3}, x_{4}\right\}$. Let $E=\{$ regular workload ( $r)$, conduct (c), field performances (g), sincerity(s), pleasing personality $(p)\}$ be the set of parameters framed to choose the best employee. Suppose the administrator $M r . X$ has the parameter set $A=\{r, c, p\} \subseteq E$ to choose the best employee. Then $f_{A}$ be the IF soft set over $X$, defined as follows:

$f(r)\left(x_{1}\right)=(0.8,0.1), f(r)\left(x_{2}\right)=(0.7,0.5), f(r)\left(x_{3}\right)=(0.9,0.1), f(r)\left(x_{4}\right)=$ $(0.7,0.2)$

$f(c)\left(x_{1}\right)=(0.6,0.2), f(c)\left(x_{2}\right)=(0.7,0.1), f(c)\left(x_{3}\right)=(0.5,0.3), f(c)\left(x_{4}\right)=(0.3,0.6)$ $f(p)\left(x_{1}\right)=(0.6,0.2), f(p)\left(x_{2}\right)=(0.7,0.1), f(p)\left(x_{3}\right)=(0.5,0.3), f(p)\left(x_{4}\right)=$ $(0.3,0.6)$

That is,

$f_{A}=(I F, A) \widetilde{\cong}\left\{F(r)=\left\{\left(x_{1}, 0.8,0.1\right),\left(x_{2}, 0.7,0.05\right),\left(x_{3}, 0.9,0.1\right),\left(x_{4}, 0.7,0.2\right)\right\}\right.$,

$F(c)=\left\{\left(x_{1}, 0.6,0.2\right),\left(x_{2}, 0.7,0.1\right),\left(x_{3}, 0.5,0.3\right),\left(x_{4}, 0.3,0.6\right)\right\}$,

$\left.F(p)=\left\{\left(x_{1}, 0.6,0.2\right),\left(x_{2}, 0.7,0.1\right),\left(x_{3}, 0.5,0.3\right),\left(x_{4}, 0.3,0.6\right)\right\}\right\}$.

The tabular representation of IF soft sets $f_{A}$ is: 


\begin{tabular}{|c|c|c|c|c|}
\hline & $x_{1}$ & $x_{2}$ & $x_{3}$ & $x_{4}$ \\
\hline$r$ & $(0.8,0.1)$ & $(0.7,0.5)$ & $(0.9,0.1)$ & $(0.7,0.2)$ \\
$c$ & $(0.6,0.2)$ & $(0.7,0.1)$ & $(0.5,0.3)$ & $(0.3,0.6)$ \\
$p$ & $(0.6,0.2)$ & $(0.7,0.1)$ & $(0.5,0.3)$ & $(0.3,0.6)$ \\
\hline
\end{tabular}

In short, we will represent $f_{A}$ as:

$f_{A} \underset{\cong}{\cong}\left\{x_{(0.8,0.1)}, x_{(0.7,0.05)}, x_{(0.9,0.1)}, x_{(0.7,0.2)}\right\},\left\{x_{(0.6,0.2)}, x_{(0.7,0.1)}, x_{(0.5,0.3)}, x_{(0.3,0.6)}\right\}$, $\left.\left\{x_{(0.6,0.2)}, x_{(0.7,0.1)}, x_{(0.5,0.3)}, x_{(, 0.3,0.6)}\right\}\right\}$.

Definition 10. [17] Two IF soft sets $f_{A}$ and $g_{B}$ over a common universe $X$, we say that $f_{A}$ is a IF soft subset of $g_{B}$, if

(1) $A \subseteq B$ and

(2) for all $a \in A, f_{a} \leq g_{a}$; implies $f_{a}$ is a IF subset of $g_{a}$.

We denote it by $f_{A} \underset{\widetilde{\subseteq}}{\widetilde{C}} g_{A} . f_{A}$ is said to be a IF soft super set of $g_{B}$, if $g_{B}$ is a IF soft subset of $f_{A}$. We denote it by $f_{A} \underset{\widetilde{\Xi}}{\widetilde{g}} g_{B}$.

Note that two IF soft sets $f_{A}$ and $g_{B}$ over a common universe $X$ are said to be IF soft equal, if $f_{A}$ is a IF soft subset of $g_{B}$ and $g_{B}$ is a If soft subset of $f_{A}$.

Definition 11. [17] The union of two IF soft sets $f_{A}$ and $g_{B}$ over the common universe $X$ is the IF soft set $h_{C}$, where $C=A \cup B$ and for all $c \in C$,

$$
h(c)=\left\{\begin{array}{cl}
f(c), & \text { if } c \in A-B \\
g(c), & \text { if } c \in B-A \\
f(c) \cup g(c), & \text { if } c \in A \cap B
\end{array}\right.
$$

We write $f_{A} \widetilde{\widetilde{\cup}} g_{B}=h_{C}$.

Definition 12. [17] The intersection $h_{C}$ of two IF soft sets $f_{A}$ and $g_{B}$ over a common universe $X$, denoted $f_{A} \widetilde{\widetilde{\cap}} g_{B}$, is defined as $C=A \cap B$, and $h(c)=f(c) \cap$ $g(c)$, for all $c \in C$.

Definition 13. [17] The relative complement of a IF soft set $f_{A}$ is the fuzzy soft set $f_{A}^{c}$, which is denoted by $\left(f_{A}\right)^{c}$ and where $f^{c}: A \rightarrow I F(X)$ is a IF set-valued function that is, for each $x \in A, f^{c}(A)$ is a IF set in $X$, whose membership function $f_{a}^{c}(x)=\left(f_{a}(x)\right)^{c}$, for all $x \in A$. Here $f_{a}^{c}$ is the membership function of $f^{c}(a)$.

Definition 14. [14] Let $\tau$ be the collection of IF soft sets over $X$, then $\tau$ is said to be a IF soft topology on $X$, if

(1) $\widetilde{\widetilde{\Phi}}_{A}, \widetilde{\widetilde{X}}_{A}$ belong to $\tau$.

(2) If $\left(f_{A}\right)_{i} \in \tau$, for all $i \in I$, then $\widetilde{\widetilde{U}}_{i \in I}\left(f_{A}\right)_{i} \in \tau$.

(3) For $f_{a}, g_{b} \in \tau$ implies that $f_{a} \widetilde{\widetilde{\cap}} g_{b} \in \tau$.

The triplet $(X, \tau, A)$ is called an IF soft topological space over $X$. Every member of $\tau$ is called IF soft open set. A IF soft set is called IF soft closed if and only if its complement is IF soft open.

Example 15. Let $X=\left\{x_{1}, x_{2}\right\}, A=\left\{e_{1}, e_{2}\right\}$ and $\tau=\left\{\widetilde{\widetilde{\Phi_{A}}}, \widetilde{\widetilde{X_{A}}}, f_{A}, g_{A}, h_{A}, k_{A}\right\}$, where $f_{A}, g_{A}, h_{A}, k_{A}$ are IF soft sets over $X$, defined as follows 


$$
\begin{aligned}
& f\left(e_{1}\right)\left(x_{1}\right)=(0.2,0.8), f\left(e_{1}\right)\left(x_{2}\right)=(0.6,0.3), \\
& f\left(e_{2}\right)\left(x_{1}\right)=(0.2,0.5), f\left(e_{2}\right)\left(x_{2}\right)=(0.9,0.1), \\
& g\left(e_{1}\right)\left(x_{1}\right)=(0.1,0.8), g\left(e_{1}\right)\left(x_{2}\right)=(0.6,0.1), \\
& g\left(e_{2}\right)\left(x_{1}\right)=(0.2,0.8), g\left(e_{2}\right)\left(x_{2}\right)=(0.8,0.1), \\
& h\left(e_{1}\right)\left(x_{1}\right)=(0.2,0.8), h\left(e_{1}\right)\left(x_{2}\right)=(0.6,0.1), \\
& h\left(e_{2}\right)\left(x_{1}\right)=(0.2,0.5), h\left(e_{2}\right)\left(x_{2}\right)=(0.9,0.1), \\
& k\left(e_{1}\right)\left(x_{1}\right)=(0.1,0.8), k\left(e_{1}\right)\left(x_{2}\right)=(0.6,0.3), \\
& k\left(e_{2}\right)\left(x_{1}\right)=(0.2,0.8), k\left(e_{2}\right)\left(x_{2}\right)=(0.8,0.1), \\
& \text { Then } \tau=\left\{\widetilde{\Phi_{A}}, \widetilde{X_{A}},\left(\left\{x_{(0.2,0.8)}, x_{(0.6,0.3)}\right\},\left\{x_{(0.2,0.5)}, x_{(0.9,0.1)}\right\}\right),\right. \\
& \left(\left\{x_{(0.1,0.8)}, x_{(0.6,0.1)}\right\},\left\{x_{(0.2,0.8)}, x_{(0.8,0.1)}\right\}\right),\left(\left\{x_{(0.2,0.8)}, x_{(0.6,0.1)}\right\},\left\{x_{(0.2,0.5)}, x_{(0.9,0.1)}\right\}\right), \\
& \left.\left(\left\{x_{(0.1,0.8)}, x_{(0.6,0.3)}\right\},\left\{x_{(0.2,0.8)}, x_{(0.8,0.1)}\right\}\right)\right\} \text { is an IF soft topology on X and hence } \\
& (X, \tau, A) \text { is an IF soft topological space over X. }
\end{aligned}
$$

Definition 16. 14] Let $\tau$ be the collection of IF soft sets over $X$. Then

(1) $\widetilde{\widetilde{\Phi}}_{A}, \widetilde{\widetilde{X}}_{A}$ are IF soft closed sets over $X$.

(2) The intersection of any number of IF soft closed sets is an IF soft closed set over $X$.

(3) The union of any two IF soft closed sets is an IF soft closed set over X.

Definition 17. 14] Let $(X, \tau, A)$ be an IF soft topological space over $X$ and $f_{A}$ be an IF soft set over $X$. Then IF soft interior of IF soft set $f_{A}$ over $X$ is denoted by $\operatorname{int}\left(f_{A}\right)$ and is defined as the union of all IF soft open sets contained in $f_{A}$. Thus $\operatorname{int}\left(f_{A}\right)$ is the largest IF soft open set contained in $f_{A}$.

Definition 18. 14 Let $(X, \tau, A)$ be an IF soft topological space over $X$ and $f_{A}$ be an IF soft set over $X$. Then the IF soft closure of $f_{A}$, denoted by $c l\left(f_{A}\right)$ is the intersection of all IF soft closed super sets of $f_{A}$. Clearly $c l\left(f_{A}\right)$ is the smallest IF soft closed set over $X$ which contains $f_{A}$.

\section{Properties of Intuitionistic Fuzz Soft Boundary}

Definition 19. 14] An IF soft set $f_{A}$ over $X$ is said to be a null IF soft set and is denoted by $\widetilde{\widetilde{\phi}}$ if and only if, for each $e \in A, f_{A}(e)=\widetilde{\widetilde{0}}$, where $\widetilde{\widetilde{0}}$ is the membership function of null IF set over $X$, which takes value $(0,1)$, for all $x \in X$.

Definition 20. 14 An IF soft set $f_{A}$ over $X$ is said to be an absolute IF soft set and is denoted by $\widetilde{\widetilde{X}}$ if and only if, for each $e \in A, f_{A}(e)=\widetilde{\widetilde{1}}$, where $\widetilde{\widetilde{1}}$ is the membership function of absolute IF set over $X$, which takes value $(1,0)$, for all $x \in X$.

Now we define:

Definition 21. The difference $h_{C}$ of two IF soft sets $f_{A}$ and $g_{B}$ over $X$, denoted by $f_{A} \tilde{\widetilde{\Upsilon}} g_{A}$, is defined as $f_{A} \widetilde{\widetilde{\}} g_{B} \approx f_{A} \widetilde{\widetilde{\cap}}\left(g_{B}\right)^{c}$. 
Example 22. Let $f_{A}$ and $g_{A}$ be two IF fuzzy soft set defined as:

$f_{A}=\left(\left\{x_{(0.2,0.8)}, x_{(0.6,0.3)}\right\},\left\{x_{(0.2,0.5)}, x_{(0.9,0.1)}\right\}\right)$ and

$g_{A} \approx\left(\widetilde{=}\left(\left\{x_{(0.1,0.8)}, x_{(0.6,0.1)}\right\},\left\{x_{(0.2,0.8)}, x_{(0.8,0.1)}\right\}\right)\right.$. Then

$f_{A} \widetilde{\widetilde{\}} g_{B} \approx f_{A} \widetilde{\widetilde{\cap}}\left(g_{B}\right)^{c}$

$\widetilde{\cong}\left(\left\{x_{(0.2,0.8)}, x_{(0.6,0.3)}\right\},\left\{x_{(0.2,0.5)}, x_{(0.9,0.1)}\right\}\right) \widetilde{\widetilde{\cap}}\left(\left\{x_{(0.8,0.1)}, x_{(0.1,0.6)}\right\},\left\{x_{(0.8,0.2)}, x_{(0.1,0.8)}\right\}\right)$

$\widetilde{\cong}\left(\left\{x_{(0.2,0.8)}, x_{(0.1,0.6)}\right\},\left\{x_{(0.2,0.5)}, x_{(0.1,0.8)}\right\}\right)$.

Definition 23. Let $(X, \tau, A)$ be an IF soft topological space over $X$ and $f_{A}$ be an IF soft set over $X$. Then the IF soft boundary of $f_{A}$, denoted by $b d\left(f_{A}\right)$ and is defined as, $b d\left(f_{A}\right) \widetilde{\approx} c l\left(f_{A}\right) \widetilde{\widetilde{n}} c l\left(\left(f_{A}\right)^{c}\right)$.

Example 24. In the above Example 15, the IF soft closed sets are

$\left.\widetilde{\widetilde{\Phi_{A}}} \widetilde{=}\left\{x_{(0,1)}, x_{(0,1)}\right\},\left\{x_{(0,1)}, x_{(0,1)}\right\}\right), \widetilde{\bar{X}_{A}} \widetilde{=}\left(\left\{x_{(1,0)}, x_{(1,0)}\right\},\left\{x_{(1,0)}, x_{(1,0)}\right\}\right)$,

$\left(\left\{x_{(0.8,0.2)}, x_{(0.3,0.6)}\right\},\left\{x_{(0.5,0.2)}, x_{(0.1,0.9)}\right\}\right),\left(\left\{x_{(0.8,0.1)}, x_{(0.1,0.6)}\right\},\left\{x_{(0.8,0.2)}, x_{(0.1,0.8)}\right\}\right)$, $\left(\left\{x_{(0.8,0.2)}, x_{(0.1,0.6)}\right\},\left\{x_{(0.5,0.2)}, x_{(0.1,0.9)}\right\}\right),\left(\left\{x_{(0.8,0.1)}, x_{(0.3,0.6)}\right\},\left\{x_{(0.8,0.2)}, x_{(0.1,0.8)}\right\}\right)$.

Let us take an IF soft set $k_{A}$ as: $k_{A} \underset{=}{\widetilde{=}}\left(\left\{x_{(0.6,0.3)}, x_{(0.1,0.8)}\right\},\left\{x_{(0.3,0.4)}, x_{(0.1,0.9)}\right\}\right)$.

Then $\operatorname{cl}\left(k_{A}\right) \stackrel{\widetilde{\cong}}{=}\left(\left\{x_{(0.8,0.2)}, x_{(0.1,0.6)}\right\},\left\{x_{(0.5,0.2)}, x_{(0.1,0.9)}\right\}\right)$. Also

$\left(k_{A}\right)^{c} \widetilde{\widetilde{\Xi}}\left(\left\{x_{(0.3,0.6)}, x_{(0.8,0.1)}\right\},\left\{x_{(0.4,0.3)}, x_{(0.9,0.1)}\right\}\right)$ and $c l\left(\left(k_{A}\right)^{c}\right) \stackrel{\widetilde{\Xi}}{\widetilde{\Phi_{A}}}$. Thus, $b d\left(k_{A}\right) \stackrel{\widetilde{\Xi}}{c l}\left(k_{A}\right) \cap \operatorname{cl}\left(\left(k_{A}\right)^{c}\right) \underset{\cong}{\widetilde{\Phi}} \widetilde{\widetilde{\Phi_{A}}}$.

Theorem 25. Let $f_{A}$ be an IF soft set of an IF soft topological space over X. Then the following hold:

(1) $\left(b d\left(f_{A}\right)\right)^{c} \stackrel{\widetilde{\Xi}}{\operatorname{int}}\left(f_{A}\right) \widetilde{\widetilde{\cup}} \operatorname{int}\left(f_{A}^{c}\right)$.

(2) $\operatorname{cl}\left(f_{A}\right) \stackrel{\widetilde{\Xi}}{\operatorname{int}}\left(f_{A}\right) \widetilde{\widetilde{\cup}} b d\left(f_{A}\right)$.

(3) $\operatorname{bd}\left(f_{A}\right) \stackrel{\widetilde{\cong}}{=} c l\left(f_{A}\right) \widetilde{\widetilde{\Upsilon}} \operatorname{int}\left(f_{A}\right)$.

(4) $\operatorname{int}\left(f_{A}\right) \stackrel{\widetilde{\approx}}{=} f_{A} \widetilde{\widetilde{\}} b d\left(f_{A}\right)$.

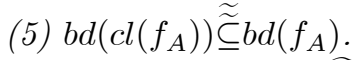

(6) $b d\left(f_{A}\right) \underset{\widetilde{\cap}}{\approx} \operatorname{int}\left(f_{A}\right) \underset{\widetilde{\widetilde{\Xi}}}{\widetilde{\widetilde{\Phi_{A}}}}$.

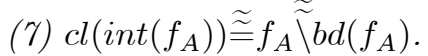

Proof. (1).

$$
\begin{aligned}
\operatorname{int}\left(f_{A}\right) \widetilde{\widetilde{U}} \operatorname{int}\left(f_{A}^{c}\right) & =\left(\left(\operatorname{int}\left(f_{A}\right)\right)^{c}\right)^{c} \widetilde{\widetilde{U}}\left(\left(\operatorname{int}\left(f_{A}^{c}\right)\right)^{c}\right)^{c} \\
& \cong\left[\left(\operatorname{int}\left(f_{A}\right)\right)^{c} \widetilde{\widetilde{\cap}} \operatorname{int}\left(f_{A}^{c}\right)^{c}\right]^{c} \\
& \cong\left[\operatorname{\cong }\left(f_{A}^{c}\right) \widetilde{\widetilde{\cap}} c l\left(f_{A}\right)\right]^{c} \\
& \cong\left(b d\left(f_{A}\right)\right)^{c} .
\end{aligned}
$$


(2).

$$
\begin{aligned}
& \operatorname{int}\left(f_{A}\right) \widetilde{\widetilde{U}} b d\left(f_{A}\right) \stackrel{\approx}{\approx} \operatorname{int}\left(f_{A}\right) \widetilde{\widetilde{\cup}}\left(\operatorname{cl}\left(f_{A}\right) \widetilde{\widetilde{n}} c l\left(f_{A}^{c}\right)\right)
\end{aligned}
$$

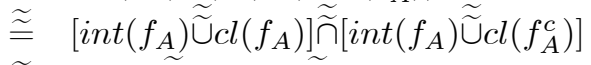

$$
\begin{aligned}
& \approx \operatorname{\cong r}\left(f_{A}\right) \widetilde{\widetilde{n}}\left[\operatorname{int}\left(f_{A}\right) \widetilde{\widetilde{U}}\left(\operatorname{int}\left(f_{A}\right)\right)^{c}\right] \\
& \approx \operatorname{\cong } c l\left(f_{A}\right) \widetilde{\widetilde{n}}\left(\operatorname{int}\left(f_{A}\right) \widetilde{\widetilde{U}}\left(\operatorname{int}\left(f_{A}\right)\right)^{c}\right) \\
& \underset{\underline{\Xi}}{\approx} l\left(f_{A}\right) \widetilde{\widetilde{\cap}} \widetilde{\widetilde{X_{A}}} \\
& \approx \operatorname{\cong r} l\left(f_{A}\right) \text {. }
\end{aligned}
$$

(3).

$$
\begin{aligned}
& b d\left(f_{A}\right) \stackrel{\approx}{\approx} c l\left(f_{A}\right) \widetilde{\widetilde{n}} c l\left(f_{A}^{c}\right) \\
& \approx c l\left(f_{A}\right) \widetilde{\widetilde{n}}\left(\operatorname{int}\left(f_{A}\right)\right)^{c} \text { (by Theorem 4.5(6)[14]). } \\
& \approx \operatorname{\cong } c l\left(f_{A}\right) \widetilde{\widetilde{\}} \operatorname{int}\left(f_{A}\right)
\end{aligned}
$$

$(4)$.

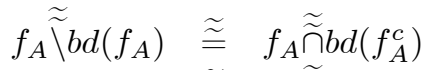

$$
\begin{aligned}
& \approx f_{A} \approx \widetilde{\widetilde{\cap}}\left(\operatorname{int}\left(f_{A}\right) \widetilde{\widetilde{\cup}} \operatorname{int}\left(f_{A}^{c}\right)\right)(b y(1)) \\
& \approx\left[f_{A} \widetilde{\widetilde{\cap}} i n t\left(f_{A}\right)\right] \widetilde{\tilde{U}}\left[f_{A} \widetilde{\widetilde{\cap}} i n t\left(f_{A}^{c}\right)\right] \\
& \underset{\cong}{\approx} \operatorname{int}\left(f_{A}\right) \widetilde{\widetilde{\cup}} \widetilde{\Phi_{A}} \\
& \stackrel{\approx}{\approx} \operatorname{int}\left(f_{A}\right) \text {. }
\end{aligned}
$$

$$
\begin{aligned}
& \operatorname{bd}\left(\operatorname{cl}\left(\left(f_{A}\right)\right) \stackrel{\approx}{\approx} \operatorname{cl}\left(\operatorname{cl}\left(f_{A}\right)\right) \underset{\widetilde{\widetilde{\imath}}}{\operatorname{int}}\left(\operatorname{cl}\left(f_{A}\right)\right)\right. \\
& \underset{\cong}{\approx} \operatorname{cl}\left(f_{A}\right) \underset{\widetilde{\widetilde{\}}}{\widetilde{\widetilde{V}}} \operatorname{int}\left(\operatorname{cl}\left(f_{A}\right)\right)
\end{aligned}
$$

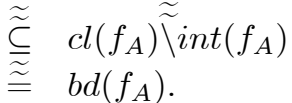

(6) follows form (3) and (7) follows directly by the definition of an IF soft boundary.

Remark 26. By (3) of above Theorem 25, it is clear that $b d\left(f_{A}\right) \widetilde{\widetilde{=}} b d\left(f_{A}^{c}\right)$.

Theorem 27. Let $f_{A}$ be an IF soft set of an IF soft topological space over X. Then:

(1) $f_{A}$ is an IF soft open set over $X$ if and only if $f_{A} \widetilde{\widetilde{\cap}} b d\left(f_{A}\right) \widetilde{\widetilde{\Xi}} \widetilde{\Phi_{A}}$.

(2) $f_{A}$ is an IF soft closed set over $X$ if and only if $b d\left(f_{A}\right) \tilde{\subseteq} f_{A}$.

(3) If $g_{A}$ be an IF soft closed(respt. open) set of an IF soft topological space with $f_{A} \widetilde{\widetilde{\subseteq}} g_{A}$, then bd( $\left.f_{A}\right) \widetilde{\widetilde{\widetilde{\subseteq}}} g_{A}\left(\right.$ respt. bd $\left.\left(f_{A}\right) \widetilde{\widetilde{\subseteq}}\left(g_{A}\right)^{c}\right)$.

Proof. (1). Let $f_{A}$ be an IF soft open set over $X$. Then $\operatorname{int}\left(f_{A}\right) \widetilde{\widetilde{=}} f_{A}$ implies $f_{A} \widetilde{\widetilde{\cap}} b d\left(f_{A}\right) \stackrel{\widetilde{\Xi}}{i n t}\left(f_{A}\right) \widetilde{\widetilde{n}} b d\left(f_{A}\right) \widetilde{\widetilde{=}} \widetilde{\widetilde{\Phi_{A}}}$.

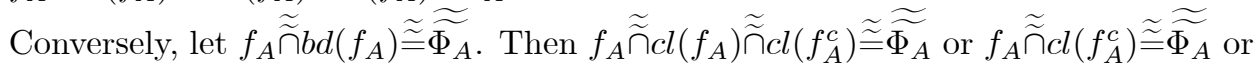


$c l\left(f_{A}^{c}\right) \tilde{\subseteq} f_{A}^{c}$, which implies $f_{A}^{c}$ is an IF soft closed and hence $f_{A}$ is an IF soft open set.

(2). Let $f_{A}$ be an IF soft closed set over $X$. Then $c l\left(f_{A}\right) \widetilde{\widetilde{=}} f_{A}$. Now

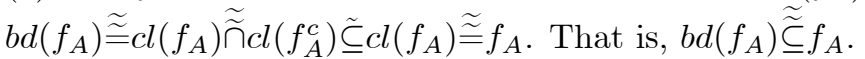

Conversely, $b d\left(f_{A}\right) \widetilde{\widetilde{\subseteq}} f_{A}$. Then $b d\left(f_{A}\right) \widetilde{\widetilde{n}} f_{A}^{c} \approx \widetilde{\widetilde{\Phi}} \widetilde{\widetilde{\Phi_{A}}}$. Since $b d\left(f_{A}\right) \widetilde{\cong} b d\left(f_{A}^{c}\right) \widetilde{\widetilde{\Xi}} \widetilde{\Phi_{A}}$, we have $b d\left(f_{A}^{c}\right) \widetilde{\widetilde{\cap}} f_{A}^{c} \approx \widetilde{\widetilde{\Xi}} \widetilde{\widetilde{\Phi_{A}}}$. By (1), $f_{A}^{c}$ is IF soft open and hence $f_{A}$ is IF soft closed.

(3). $f_{A} \underset{\widetilde{\widetilde{\subseteq}}}{\widetilde{c}} g_{A}$ follows that $c l\left(f_{A}\right) \widetilde{\widetilde{\widetilde{c}}} c l\left(g_{A}\right)$. Since $g_{A}$ is IF soft closed, then we get,

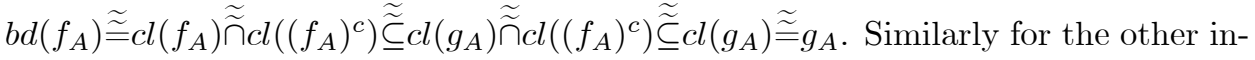
clusion.

The following example shows that (1) and (2) are not true, if $f_{A}$ is not IF soft open and IF soft closed respectively.

Example 28. In the above Example 15, an IF soft closed sets are $\left.\widetilde{\Phi_{A}} \widetilde{=}\left\{x_{(0,1)}, x_{(0,1)}\right\},\left\{x_{(0,1)}, x_{(0,1)}\right\}\right), \widetilde{X_{A}} \approx\left(=\left(x_{(1,0)}, x_{(1,0)}\right\},\left\{x_{(1,0)}, x_{(1,0)}\right\}\right)$, $\left(\left\{x_{(0.8,0.2)}, x_{(0.3,0.6)}\right\},\left\{x_{(0.5,0.2)}, x_{(0.1,0.9)}\right\}\right),\left(\left\{x_{(0.8,0.1)}, x_{(0.1,0.6)}\right\},\left\{x_{(0.8,0.2)}, x_{(0.1,0.8)}\right\}\right)$, $\left(\left\{x_{(0.8,0.2)}, x_{(0.1,0.6)}\right\},\left\{x_{(0.5,0.2)}, x_{(0.1,0.9)}\right\}\right),\left(\left\{x_{(0.8,0.1)}, x_{(0.3,0.6)}\right\},\left\{x_{(0.8,0.2)}, x_{(0.1,0.8)}\right\}\right)$. Let us take $f_{A} \underset{\cong}{\widetilde{=}}\left(\left\{x_{(0.6,0.1)}, x_{(0.1,0.7)}\right\},\left\{x_{(0.7,0.3)}, x_{(0.1,0.9)}\right\}\right)$, which is not IF soft open and not IF soft closed. Then $\operatorname{cl}\left(f_{A}\right) \widetilde{\widetilde{=}}\left(\left\{x_{(0.8,0.1)}, x_{(0.1,0.6)}\right\},\left\{x_{(0.8,0.2)}, x_{(0.1,0.8)}\right\}\right)$. Also $\left(f_{A}\right)^{c} \widetilde{\cong}\left(\left\{x_{(0.1,0.6)}, x_{(0.7,0.1)}\right\},\left\{x_{(0.3,0.7)}, x_{(0.9,0.1)}\right\}\right)$ and $c l\left(\left(f_{A}\right)^{c}\right) \stackrel{\widetilde{\cong}}{\widetilde{\widetilde{X}}}$. Thus, $b d\left(f_{A}\right) \widetilde{\cong} c l\left(f_{A}\right) \cap c l\left(\left(f_{A}\right)^{c}\right) \widetilde{\cong}\left(\left\{x_{(0.8,0.1)}, x_{(0.1,0.6)}\right\},\left\{x_{(0.8,0.2)}, x_{(0.1,0.8)}\right\}\right)$. We observe that, $f_{A} \widetilde{\widetilde{\cap}} b d\left(f_{A}\right) \widetilde{\widetilde{\neq}} \widetilde{\widetilde{\Phi_{A}}}$ and bd $\left(f_{A}\right) \tilde{ \pm} f_{A}$.

The following example verify (3) of above Theorem 27.

Example 29. In the above Example, let us take an IF fuzzy soft closed set $g_{A} \widetilde{\cong}\left(\left\{x_{(0.8,0.1)}, x_{(0.3,0.6)}\right\},\left\{x_{(0.8,0.2)}, x_{(0.1,0.8)}\right\}\right)$ and any IF soft set $f_{A} \approx{ } \widetilde{=}\left(\left\{x_{(0.6,0.1)}, x_{(0.1,0.7)}\right\},\left\{x_{(0.7,0.3)}, x_{(0.1,0.9)}\right\}\right)$. Then $f_{A} \widetilde{\widetilde{\subseteq}} g_{A}$. Clearly, $b d\left(f_{A}\right) \widetilde{\widetilde{\Xi}}\left(\left\{x_{(0.8,0.1)}, x_{(0.1,0.6)}\right\},\left\{x_{(0.8,0.2)}, x_{(0.1,0.8)}\right\}\right) \widetilde{\widetilde{\subseteq}} g_{A}$.

Theorem 30. Let $f_{A}$ and $g_{B}$ be an IF soft sets of an IF soft topological space over $X$. Then the following hold:

(1) $b d\left(\left[f_{A} \widetilde{\widetilde{\cup}} g_{B}\right]\right) \underset{\widetilde{\widetilde{c}}}{\widetilde{\widetilde{c}}}\left[b d\left(f_{A} \widetilde{\widetilde{\cap}}\left(g_{B}^{c}\right)\right)\right] \widetilde{\widetilde{\cup}}\left[b d\left(g_{B}\right) \widetilde{\widetilde{n}} c l\left(\left(\left(f_{A}\right)^{c}\right)\right)\right]$.

(2) $b d\left(\left[f_{A} \widetilde{\widetilde{\cap}} g_{B}\right]\right) \widetilde{\widetilde{\widetilde{C}}}\left[b d\left(f_{A}\right) \widetilde{\widetilde{n}} c l\left(g_{B}\right)\right] \widetilde{\widetilde{\cup}}\left[b d\left(g_{B}\right) \widetilde{\widetilde{n}} c l\left(\left(f_{A}\right)\right)\right]$. 
Proof. (1).

$$
\begin{aligned}
& b d\left(\left(f_{A} \widetilde{\widetilde{\cup}} g_{B}\right)\right) \stackrel{\cong}{\cong} l\left(\left(f_{A} \widetilde{\widetilde{\cup}} g_{B}\right)\right) \widetilde{\widetilde{\cap}} c l\left(\left(\left(f_{A} \widetilde{\widetilde{\cup}} g_{B}\right)^{c}\right)\right) \\
& \underset{\cong}{\approx}\left(c l\left(f_{A}\right) \widetilde{\widetilde{U}} c l\left(g_{B}\right)\right) \widetilde{\widetilde{n}} c l\left(\left(f_{A}^{c} \widetilde{\widetilde{\cap}} g_{B}^{c}\right)\right)
\end{aligned}
$$

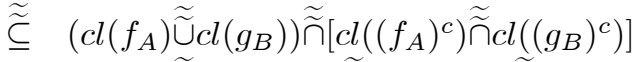

$$
\begin{aligned}
& \underset{\cong}{\cong}\left(c l\left(f_{A}\right) \widetilde{\widetilde{n}} c l\left(\left(f_{A}\right)^{c}\right)\right) \widetilde{\widetilde{n}}\left(c l\left(\left(g_{B}\right)^{c}\right) \widetilde{\widetilde{U}} c l\left(g_{B}\right)\right) \widetilde{\widetilde{n}}\left[\operatorname{cl}\left(\left(f_{A}\right)^{c}\right) \widetilde{\widetilde{n}} c l\left(\left(g_{B}\right)^{c}\right)\right] \\
& \underset{\widetilde{\widetilde{ }}}{\widetilde{\sigma}}\left(b d\left(f_{A}\right) \widetilde{\widetilde{n}} c l\left(\left(g_{B}\right)^{c}\right)\right) \widetilde{\widetilde{\cup}}\left(b d\left(g_{B}\right) \widetilde{\widetilde{n}} c l\left(f_{A}^{c}\right)\right) \\
& \widetilde{\widetilde{\subseteq}} b d\left(f_{A}\right) \widetilde{\widetilde{\cup}} b d\left(g_{B}\right) \text {. }
\end{aligned}
$$

(2).

$$
\begin{aligned}
& b d\left(\left[f_{A} \widetilde{\widetilde{\cap}} g_{B}\right]\right) \underset{\cong}{\approx} \operatorname{cl}\left(\left(f_{A} \widetilde{\widetilde{\cap}} g_{B}\right)\right) \widetilde{\widetilde{\cap}} c l\left(\left(f_{A} \widetilde{\widetilde{\cap}} g_{B}\right)^{c}\right)
\end{aligned}
$$

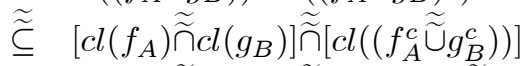

$$
\begin{aligned}
& =\left[\operatorname{cl}\left(f_{A}\right) \widetilde{\tilde{n}} c l\left(g_{B}\right)\right] \widetilde{\tilde{n}}\left[c l\left(\left(f_{A}\right)^{c}\right) \widetilde{\widetilde{U}} c l\left(\left(g_{B}\right)^{c}\right)\right] \\
& \approx\left[\left(\cos \left(f_{A}\right) \widetilde{\widetilde{n}} c l\left(\left(g_{B}\right)\right)\right) \widetilde{\widetilde{n}} c l\left(\left(f_{A}\right)^{c}\right)\right] \widetilde{\widetilde{U}}\left[\left(\operatorname{cl}\left(\left(f_{A}\right)\right) \widetilde{\widetilde{n}} c l\left(\left(g_{B}\right)\right)\right) \widetilde{\widetilde{n}} c l\left(\left(g_{B}\right)^{c}\right)\right] \\
& \widetilde{\cong}\left(b d\left(\left(f_{A}\right)\right) \widetilde{\widetilde{n}} b d\left(\left(g_{B}\right)\right)\right) \widetilde{\widetilde{U}}\left(c l\left(f_{A}\right) \widetilde{\widetilde{n}} b d\left(g_{B}\right)\right) \text {. }
\end{aligned}
$$

Corollary 31. Let $f_{A}$ and $g_{B}$ be IF soft sets of an IF soft topological space over

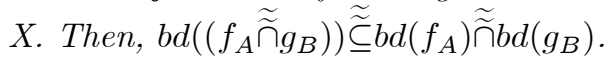

Theorem 32. Let $f_{A}$ be an IF soft set of an IF soft topological space over $X$. Then we have: $b d\left(\left(b d\left(\left(b d\left(f_{A}\right)\right)\right)\right)\right) \widetilde{\cong} b d\left(\left(b d\left(f_{A}\right)\right)\right)$.

Proof.

$$
\begin{aligned}
b d\left(\left(b d\left(\left(b d\left(f_{A}\right)\right)\right)\right)\right) & \approx \\
= & c l\left(\left(b d\left(\left(b d\left(f_{A}\right)\right)\right)\right)\right) \widetilde{\widetilde{\cap}} c l\left(\left(\left(b d\left(\left(b d\left(f_{A}\right)\right)\right)\right)^{c}\right)\right) \\
& \left.\left.=\left(b d\left(\left(f_{A}\right)\right)\right)\right)\right)
\end{aligned}
$$

Now consider

$$
\begin{aligned}
& \left(\left(b d\left(\left(b d\left(f_{A}\right)\right)\right)\right)^{c}\right) \stackrel{\approx}{\approx}\left[c l\left(\left(b d\left(f_{A}\right)\right)\right) \widetilde{\widetilde{\cap}}\left(\left(b d\left(f_{A}\right)\right)^{c}\right)\right]^{c} \\
& \approx\left[\quad\left[b d\left(f_{A}\right) \widetilde{\cap} c l\left(\left(b d\left(f_{A}\right)\right)^{c}\right)\right]^{c}\right. \\
& \approx\left(b d\left(f_{A}\right)\right)^{c} \widetilde{\widetilde{U}}\left(c l\left(\left(b d\left(f_{A}\right)\right)^{c}\right)\right)^{c}
\end{aligned}
$$

Therefore

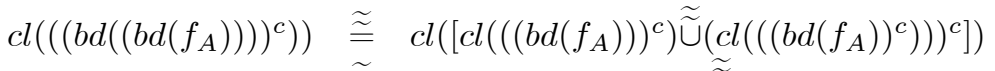

$$
\begin{aligned}
& \approx \operatorname{\approx } c l\left(\left(c l\left(\left(\left(b d\left(f_{A}\right)\right)\right)^{c}\right)\right)\right) \widetilde{\widetilde{U}} c l\left(\left(\left(\operatorname{cl}\left(\left(\left(b d\left(f_{A}\right)\right)^{c}\right)\right)\right)^{c}\right)\right) \\
& \left.\approx g_{A} \widetilde{\widetilde{\cup}}\left(\left(c l\left(\left(\left(b d\left(g_{A}\right)\right)\right)^{c}\right)\right)\right)^{c}\right) \widetilde{\cong} \widetilde{\widetilde{X_{A}}}
\end{aligned}
$$

where $g_{A} \widetilde{\widetilde{=}} c l\left(\left(\operatorname{cl}\left(\left(\left(b d\left(f_{A}\right)\right)\right)^{c}\right)\right)\right)$. From $(1)$ and $(2)$, we have

$$
b d\left(\left(b d\left(\left(b d\left(f_{A}\right)\right)\right)\right)\right) \widetilde{\cong} b d\left(\left(b d\left(f_{A}\right)\right)\right) \widetilde{\widetilde{n}} \widetilde{\widetilde{X_{A}}} \widetilde{\underline{\Xi}} b d\left(\left(b d\left(f_{A}\right)\right)\right) \text {. }
$$


Theorem 33. Let $f_{A}$ and $g_{A}$ be a IF soft open sets of IF soft topological space over $X$. Then the following hold:

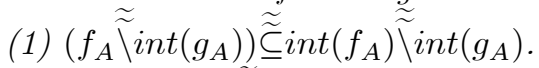

(2) $b d\left(\operatorname{int}\left(f_{A}\right)\right) \widetilde{\widetilde{\subseteq}} b d\left(f_{A}\right)$.

Proof. (1).

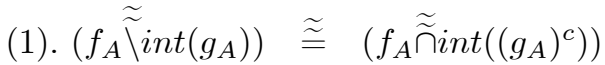

$$
\begin{aligned}
& \approx \operatorname{int}\left(f_{A}\right) \widetilde{\widetilde{n}} \operatorname{int}\left(\left(g_{A}\right)^{c}\right)
\end{aligned}
$$

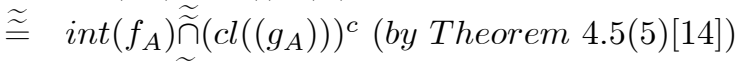

$$
\begin{aligned}
& \approx \operatorname{sint}\left(f_{A}\right) \widetilde{\widetilde{\}} c l\left(g_{A}\right) \\
& \widetilde{\widetilde{\subseteq}} \operatorname{int}\left(f_{A}\right) \widetilde{\widetilde{\}} \operatorname{int}\left(g_{A}\right) \text {. }
\end{aligned}
$$

(2).

$$
\begin{aligned}
& \text { (2). } b d\left(\operatorname{int}\left(f_{A}\right)\right) \underset{\widetilde{\approx}}{\widetilde{\approx}} \operatorname{cl}\left(\operatorname{int}\left(f_{A}\right)\right) \widetilde{\widetilde{n}} c l\left(\left(\left(\operatorname{int}\left(f_{A}\right)\right)^{c}\right)\right) \\
& \underset{\widetilde{\subseteq}}{\widetilde{\tau}} \operatorname{cl}\left(\operatorname{int}\left(f_{A}\right)\right) \widetilde{\widetilde{n}} c l\left(\left(c l\left(\left(f_{A}^{c}\right)\right)\right)\right) \text { (by Theorem 4.5(5)[14]) } \\
& \widetilde{\widetilde{\subseteq}} c l\left(f_{A}\right) \widetilde{\widetilde{n}} c l\left(\left(f_{A}^{c}\right)\right) \widetilde{\widetilde{\Xi}} b d\left(f_{A}\right) \text {. }
\end{aligned}
$$

Theorem 34. Let $f_{A}$ be an IF soft set of an IF soft topological space over X. Then $b d\left(f_{A}\right) \stackrel{\widetilde{\Xi}}{\widetilde{\Phi_{A}}}$ if and only if $f_{A}$ is an IF soft closed set and an IF soft open set.

Proof. Suppose that $b d\left(f_{A}\right) \stackrel{\widetilde{=}}{\widetilde{\Phi}}$.

(i) First we prove that $f_{A}$ is an IF soft closed set. Consider

$$
\begin{aligned}
& b d\left(f_{A}\right) \stackrel{\widetilde{\Xi} \widetilde{\Phi_{A}}}{\widetilde{\sigma}} \operatorname{cl}\left(f_{A}\right) \widetilde{\widetilde{n}} c l\left(\left(f_{A}^{c}\right)\right) \underset{\widetilde{=}}{\widetilde{\Phi_{A}}} \\
& \Rightarrow \operatorname{cl}\left(f_{A}\right) \underset{\widetilde{\widetilde{C}}}{\widetilde{\widetilde{C}}}\left(\mathrm{cl}\left(\left(f_{A}^{c}\right)\right)\right)^{c} \underset{\underline{\Xi}}{\operatorname{int}}\left(f_{A}\right) \text { (by Theorem 4.5(6)[14]) }
\end{aligned}
$$

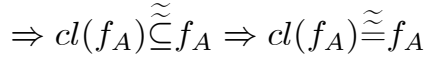

This implies that $f_{A}$ is an IF soft closed set.

(ii) Using (i), we now prove that $f_{A}$ is an IF soft open set.

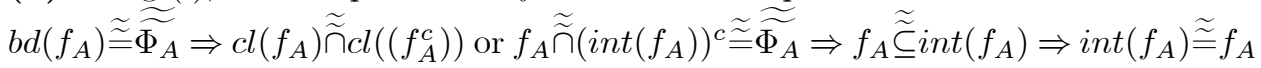
This implies that $f_{A}$ is an IF soft open set.

Conversely, suppose that $f_{A}$ is an IF soft open and an IF soft closed set. Then

$$
\begin{aligned}
& b d\left(f_{A}\right) \stackrel{\cong}{\approx} c l\left(f_{A}\right) \widetilde{\widetilde{n}} c l\left(\left(f_{A}^{c}\right)\right) \\
& \approx c l\left(f_{A}\right) \widetilde{\widetilde{n}}\left(\operatorname{int}\left(f_{A}\right)\right) c(\text { by Theorem 4.5(6)[14] } \\
& \approx f_{A} \widetilde{\widetilde{n}} f_{A}^{c} \approx \widetilde{\cong} \widetilde{\Phi_{A}} \text {. }
\end{aligned}
$$

This completes the proof. 
The following example shows that the condition that $f_{A}$ is IF soft open and IF soft closed is necessary in the above theorem.

Example 35. In the above Example 28, let us take an IF soft set $\left.\left.f_{A} \approx{ }_{(0.7,0.1)}, x_{(0.1,0.6)}\right\},\left\{x_{(0.6,0.5)}, x_{(0.1,0.9)}\right\}\right)$., which is not IF soft closed and IF soft open. Calculations show that

$b d\left(f_{A}\right) \widetilde{\cong} c l\left(f_{A}\right) \cap \operatorname{cl}\left(\left(f_{A}\right)^{c}\right) \widetilde{\widetilde{\Xi}}\left(\left\{x_{(0.8,0.1)}, x_{(0.1,0.6)}\right\},\left\{x_{(0.8,0.2)}, x_{(0.1,0.8)}\right\}\right) \widetilde{\widetilde{\neq}} \widetilde{\widetilde{\Phi_{A}}}$.

\section{Conclusion}

The importance of decision making problem in an imprecise environment is growing very significantly in recent years. The concept of intuitionistic fuzzy soft sets in a decision making problem and the problem is solved with the help of 'similarity measurement' technique. In this paper, we initiated the concept of IF soft boundary. We discussed and explored the characterizations and properties of IF soft boundary in general as well as in terms of IF soft interior and IF soft closure. Examples and counter examples are also presented to validate the discussed results. In future studies, we will study the further topological structures in IF soft sets. We will also explore applications of the topological structures of IF soft sets in medical diagnosis system, and other decision making problems. We hope that the addition of this concept and properties will be a good addition in the tool box of IF soft sets and will be helpful for the researchers working in this field.

Acknowledgement. Author is thankful to the anonymous reviewers for their beneficial comments towards the improvement of the paper.

\section{REFERENCES}

[1] Atanassov, K., lntuitionistic fuzzy sets, in: V. Sgurev, Ed., VII ITKR's Session, Sofia, June 1983, Central Sci. and Techn. Library, Bulg. Academy of Sciences, 1984.

[2] Atanassov, K., lntuitionistic fuzzy sets, Fuzzy Sets and Systems, 20 (1986), 87-96.

[3] Atanassov, K. and Stoeva S., Intuitionistic fuzzy sets, Polish Symp. on Interval and Fuzzy Mathematics, Poznan, (August 1983), 23-26.

[4] Chen, D., Tsang, E. C. C., Yeung, D. S., Wang, X., The parameterization reduction of soft sets and its applications, Comput. Math. Appl., 49 (2005), 757-763.

[5] Coker, D., An introduction to intuitionistic fuzzy topological spaces, Fuzzy Sets and Systems, 88 (1997), 81-89.

[6] Feng, F., Jun, Y. B., Liu, X., Li, L., An adjustable approach to fuzzy soft set based decision making, J. Comput. Appl. Math., 234 (1) (2010), 10-20.

[7] Hussain, S., A note on soft connectedness, Journal of Egyptian Mathematical Society, 23(1) (2015), 6-11.

[8] Hussain, S., Ahmad, B., Some properties of soft topological spaces, Computers and Mathematics with Applications, 62(11) (2011), 4058-4067.

[9] Hussain, S., Ahmad B., Soft separation axioms in soft topological spaces, Hacettepe Journal of Mathematics and Statistics, 44(3) (2015), 559-568.

[10] Hussain, S., On some soft functions, Mathematical Science Letters, 4(1)(2015), 55-61. 
[11] Hussain, S., On some generalized soft mappings, Hacettepe Journal of Mathematics and Statistics, 45(3) (2016), 743-754.

[12] Hussain, S., Akiz, H. F., Alajlan, A. I., On algebraic properties of soft real points, Moroccan J. of Pure and Appl. Anal. (MJPAA), 4(1) (2018) 9-16.

[13] Hussain, S., Akiz, H. F., Alajlan, A. I., On soft real point matrices and their operations, Fixed Point Theory and Applications, 9 (2019), 1-20.

[14] Li, Z., Cui, R., On the topological structures of intuitionistic fuzzy soft sets, Annals of fuzzy Mathematics and Informatics, 5(1) (2013), 229-239.

[15] Maji, P. K., Biswas, R., Roy, A. R., Fuzzy soft sets, J. Fuzzy Math., 9(3)(2001),589-602.

[16] Maji, P. K., Biswas, R., Roy, A. R., Intuitionistic fuzzy soft sets, The Journal of Fuzzy Mathematics, 9(3) (2001), 677-691.

[17] Maji, P. K., Biswas, R., Roy, A. R., On Intuitionistic fuzzy soft sets, The Journal of Fuzzy Mathematics, 12(3) (2004), 669-683.

[18] Maji, P. K., More on intuitionistic fuzzy soft sets, LNCS, 5908,(2006), 231-240.

[19] Molodtsov, D., Soft set theory: first results, Computers and Mathematics with Applications, 37 (1999)19-31.

[20] Roy, A. R., Maji, P. K., A fuzzy soft set theoretic approach to decision making problems, $J$. Comput. Appl. Math., 203 (2007), 412-418.

[21] Xiao, Z., Gong, K., Zou, Y., A combined forecasting approach based on fuzzy soft sets, J. Comput. Appl. Math., 228 (1) (2009), 326-333.

[22] Yin, Y., Li, H., Jun, Y. B., On algebraic structure of intuitionistic fuzzy soft sets, Computers and Mathematics with Applications, 64 (2012), 2896-2911.

[23] Zadeh, L. A., Fuzzy sets, Information and Control, 8(1996), 338-353.

[24] Zou, Y., Xiao, Z., Data analysis approaches of soft sets under incomplete information, Knowledge-Based Syst., 21(8) (2008), 941-945. 